\title{
Ultrasound practice in emergency medicine
}

\author{
Ali Haydar Akça \\ Department of Emergency Medicine, Van Training and Research Hospital, Van
}

\begin{abstract}
The use of ultrasound in emergency department has dramatically increased in recent years. Performing ultrasound examination without the aid of a radiologist at the patient's bedside has become an usual experience for the emergency physicians and is now taking place in residency training. Ultrasound use has an important place in patient management especially in critically ill and undifferentiated patients. It has the capacity to revolutionize patient care and improve diagnostic and procedural efficacy. Access to the ultrasound has to be ensured in the emergency departments in order to increase patient safety and fasten the definite diagnosis and treatment.
\end{abstract}

Key Words: Ultrasound, emergency, diagnosis

\section{Introduction}

Ultrasound is a safe, non-invasive, repeatable portable imaging modality, applicable to many areas of emergency medicine. So on, the use of ultrasound in emergency department has dramatically increased in recent years. American College of Emergency Physicians (ACEP) published a position statement on ultrasound, for the first time in 1990, indicating that "emergency ultrasonography should be performed by an appropriately trained and credentialed physician including emergency physicians" (1). Since that up today performing ultrasound examination without the aid of a radiologist at the patient's bedside has become an usual experience for the emergency physicians and is now taking place in residency training $(2,3)$. Improved patient safety and decreased time to definitive care are the main drivers of this expansion (4-6).

Ultrasonography (USG) is undergoing great changes. Very small ultrasound units are being developed. Some people feel that ultrasound is the future stethoscope and should be available on an emergency basis at all times. Advances in ultrasound technology have fueled the emergence of ultrasound, including improved ease of use, superior image quality and lower-cost USG units. Although ionizing radiation from computed tomography (CT) scanning is increasingly recognized as a potentially major cause of cancer, ultrasonography has been used in obstetrics for decades, with no epidemiologic evidence of harmful effects at normal diagnostic levels (7).

Point-of-care (POC) ultrasound refers to the use of ultrasonography at patient's bedside for diagnostic and therapeutic purposes. It differs from comprehensive ultrasound exam in several aspects. POC exams are generally employed to detect acute, potentially life-threatening conditions where detection at the bedside expedites patient care. POC ultrasound examination has to be performed for a well-defined, focused and goal-directed purpose linked to improving patient outcomes at the patient's bedside. It has to be easily learned and quickly performed and findings are easily recognizable (5).

Ultrasound use has an important place in patient management especially in critically ill and undifferentiated patients. It has the capacity to revolutionize patient care and improve diagnostic and procedural efficacy, decrease complications and limit pain and suffering. Conditions requiring immediate (within 15 to 20 minutes) ultrasound evaluation include expanding or leaking abdominal aneurysm, suspected ectopic pregnancy, and traumatic hemoperitoneum. When patients with these conditions are unstable, they require even more rapid imaging in the operating room.

Some of usage areas of ultrasound in emergency medicine are listed below:

- Focused Assessment with Sonography in Trauma (FAST): FAST is a modality to rapidly detect free fluid (usually blood) in the peritoneal, pericardial, or pleural spaces in trauma patients (8). This technique provides repeatable, low-cost and sensitive evaluation of trauma patients without radiation.

- USG-guided interventions: Emergency procedures like central venous catheterization or pericardiocentesis with lower complication rates 
and decreased time for procedure. Strong evidence exists supporting the use of ultrasound guidance for central venous catheter placement compared with traditional landmark techniques, including limited support for its use in the emergency department (9-11).

- Undifferentiated shock patients: Shock etiologies can be distinguished with assessing heart, intravascular volume and major artery and veins as hypovolemic, distributive, cardiogenic or obstructive with the aid of ultrasound (12).

- Abdominal pain: Ultrasound may give us crucial information about etiology of abdominal pain in hepatic or gallbladder diseases, aortic pathologies etc.

- Deep vein thrombosis (DVT): 2-point compression testing is reliable in selected patients (no chronic or recurrent DVTs, no obese patients).

Ultrasound should be used for other diagnoses like pneumothorax, pulmonary thromboembolism, foreign bodies in soft tisseus and also for other purposes like endotracheal tube placement confirmation.

In conclusion; point of care ultrasound in emergency medicine practice is getting wider everyday with many advantages. Emergency ultrasound is part of the essential training requirement for emergency medicine programs in the United States and elsewhere $(13,14)$. Also ultrasound trainings for emergency physicians are readily accessible in our country. Key considerations to improve efficacy and quality of point of care ultrasound exams for different clinical applications include optimization of provider training, patient factors and ultrasound equipment features. As the experiences augmented, it will affect patient safety in a positive way. To provide it, access to the ultrasound has to be ensured in the emergency departments.

\section{References}

1. American College of Emergency Physicians (1990) Council resolution on ultrasound. ACEP News, November 1990

2. Emergency ultrasound guidelines. Ann Emerg Med. 2009; 53: 550-570.
3. Lewiss RE, Pearl M, Nomura JT, et al. Cord-aeus: Consensus document for the emergency ultrasound milestone project. Acad Emerg Med 2013; 20: 740745.

4. Jones AE, Tayal VS, Sullivan DM, Kline JA. Randomized, controlled trial of immediate versus delayed goal-directed ultrasound to identify the cause of nontraumatic hypotension in emergency department patients. Crit Care Med 2004; 32: 17031708.

5. Melniker LA, Leibner E, McKenney MG, et al. Randomized controlled clinical trial of point-ofcare, limited ultrasonography for trauma in the emergency department: The first sonography outcomes assessment program trial. Ann Emerg Med 2006; 48: 227-235.

6. Shekelle PG, Wachter RM, Pronovost PJ, et al. Making health care safer ii: An updated critical analysis of the evidence for patient safety practices. Evid Rep Technol Assess 2013: 1-945.

7. Moore CL, Copel JA. Point-of-care ultrasonography. N Engl J Med 2011; 364: 749-757.

8. Sheng AY, Dalziel P, Liteplo AS, Fagenholz P, Noble VE. Focused assessment with sonography in trauma and abdominal computed tomography utilization in adult trauma patients: Trends over the last decade. Emerg Med Int 2013: 678380.

9. Hind D, Calvert N, McWilliams R, et al. Ultrasonic locating devices for central venous cannulation: meta-analysis. BMJ 2003; 327: 361.

10. Leung J, Duffy M, Finckh A. Real-time ultrasonographically-guided internal jugular vein catheterization in the emergency department increases success rates and reduces complications: a randomized, prospective study. Ann Emerg Med 2006; 48: 540-547.

11. Fragou M, Gravvanis A, Dimitriou V, et al. Realtime ultrasound-guided subclavian vein cannulation versus the landmark method in critical care patients: a prospective randomized study. Crit Care Med 2011; 39: 1607-1612.

12. Perera P, Mailhot T, Riley D, Mandavia D. The RUSH exam: Rapid Ultrasound in SHock in the evaluation of the critically 1ll. Emerg Med Clin North Am 2010; 28: 29-56.

13. American College of Emergency Physicians (ACEP) Emergency ultrasound Guidelines. Am Coll Emerg Physicians 2008; 53: 1-38.

14. Sippel S, Muruganandan K, Levine A, Shah S. Review article: Use of ultrasound in the developing world. Int J Emerg Med 2011; 4: 72. 